\title{
A Study on Color Image Enhancement Technique of Fusion using Automated Histogram Specification
}

\author{
S. Gopinathan, PhD \\ Dept. of Computer Science \\ University of Madras \\ Chennai, India
}

\author{
S. Yamini \\ Dept. of Computer Science \\ University of Madras \\ Chennai, India
}

\begin{abstract}
The Image enhancement techniques is to improve a characteristic or quality of an image such that the resulting image is better than the original. The color image enhancement methodology uses an automated way of target Image generation is Proposed here. The method is a simple color image enhancement technique where the range(the gamut) of R,G,B channels is optimally preserved. Target image with highest histogram correlation are used for image fusion. The maximum possible Enhancement is achieved using a Hyperbolic tangent activation function or tanh. The proposed method has been compared with Raji at el method given in the literature. It is a new quantitative validation approach to identify visibility loss problem that may occur during enhancement.
\end{abstract}

\section{General Terms}

Image Enhancement

\section{Keywords}

Enhancement, Target Image, Fusion, visibility loss.

\section{INTRODUCTION}

The Image enhancement is used to improve the quality of an image for visual perception of human beings. Contrast is an important factor in any individual estimation of image quality[3]. Contrast Enhancement is a process that allows images features to show up more visibly by making best use of the colors presented on the display device[5]. Various enhancement methods have been proposed in the literature. This primarily includes histogram processing methods, graylevel compression, stretching using exponentials,

polynomials ,spatial statistical filtering and frequency domain processing techniques[8].Histogram Equalization[HE][14] is one of the most well-known methods for contrast enhancement. It is useful for images with poor intensity distribution. Moreover it cannot be applied directly to color images, because HE Applied to each color channel independently disturbs perceived colorfulness of the obtained image. Most of the existing techniques are intended only for certain category of images.

These are all well known algorithms that independently handle gamut problem effectively but lack capability of handle loss of visibility problem. Visibility[14] is a measure of how clearly an image can be viewed and it is an essential property which has to be improved after enhancement. Each computed parameter of input image is compared against stored vector by computing Euclidean distance. When an input image is given it can be compared against images in database to retrieve highly correlated set of images. The statistical parameter which results in minimum Euclidean distance[14] is taken as the criteria for selecting images for fusion. Among the obtained set of fused images, one with highest entropy is selected as target image. EME is defined as the measure of enhancement[6] or the measure of improvement. EME of target image is compared with EME of input image to ensure success of enhancement. It was observed that if the EME[2] of target image is lower compared to EME of input image, histogram specification can lead to loss of visibility.

\section{RELATED WORK}

S.Jayaraman,S.Esakkirajan and T.Veerakumar had discussed about the topics on Digital Image Processing including Image Enhancement ,Image fusion, Histogram Specification etc..., in a detailed manner.

Rafel C.Gonzale and Richard E.Woods had discussed about the introduction to Digital Image processing For beginners and also explained about the topics on image Enhancement and Image fusion in a detailed manner.

T.zhang,R.Ramakrishnan and M.Livny,BIRCH had discussed about A New Data Clustering Algorithm and its Applications,Data Mining and Knowledge Discovery in a detailed manner.

Agaian Sos S,Karen panetta and Artyom M.Grigoryan, had discussed about A new measure of image enhancement,in IASTED International Conference on Signal Processing \& Communication in a detailed manner.

S.F.Naik and C.A.Murthy, had discussed about Huepreserving color image enhancement without gamut problem,IEEE Trans Image process in a detailed manner.

Avcibas,N.Memon and B.Sankur had discussed about Steganalysis using Image Quality Metrics,IEEE Transactions on Image processing in a detailed manner.

Hassan Naglaa, and Norio Akamatsu had discussed about A New Approach for Contrast Enhancement Using Sigmoid Function,The International Arab Journal of Information Technology in a detailed manner.

S.K.Saha,A.K.Das,B.Chanda had discussed about CBIR using perception based Texture and Color Measures,Pattern Recognition in a detailed manner.

M.Grundland,N.A.Dodgson had discussed about Colorhistogram specification by histogram warping in a detailed manner.

Iyad Jafar Hao Ying had discussed about A New Method For Image Constrast Enhancement Based on Automatic Specification of local Histogram in a detailed manner.

Rafael C.Gonzalez,Richard E.Woods had discussed about Digital Image Processing in a detailed manner.

Pavithra P,Ramyashree N,Shruthi T.V and Jharna Majumdar had discussed about Image Enhancement by Histogram Specification Using Multiple Target Images in a detailed manner. 
Kalyan Roy and Joydeep Mukherjee had discussed about Image Similarity Measure using Color Histogram,Color Coherence Vector, and Sobel Method in a detailed manner.

M.Nikolova had discussed about A fast algorithm for exact histogram specification Simple extension to colour images, in SCALE Space and Variational Methods in Computer Vision in a detailed manner.

Codruta Orniana Ancuti and Cosmin Ancuti had discussed about Single Image Dehazing by Multi-Scale Fusion,IEEE Transactions on Image Processing in a detailed manner.

\section{PROPOSED FHN METHOD}

The proposed Fusion, Histogram Specification, Neural Networks [FHN] method is a color image enhancement technique that adaptively generates the good quality target image for different categories of input image. The method finds a suitable target from image database for image fusion. The image database that we use in our work contains randomly selected set of images of distinct categories. This enables the algorithm in finding the most similar images for fusion such that it would lead to generation of a good target image. Better the target image selected better would be the level of enhancement achieved.

\section{BLOCK DIAGRAM}

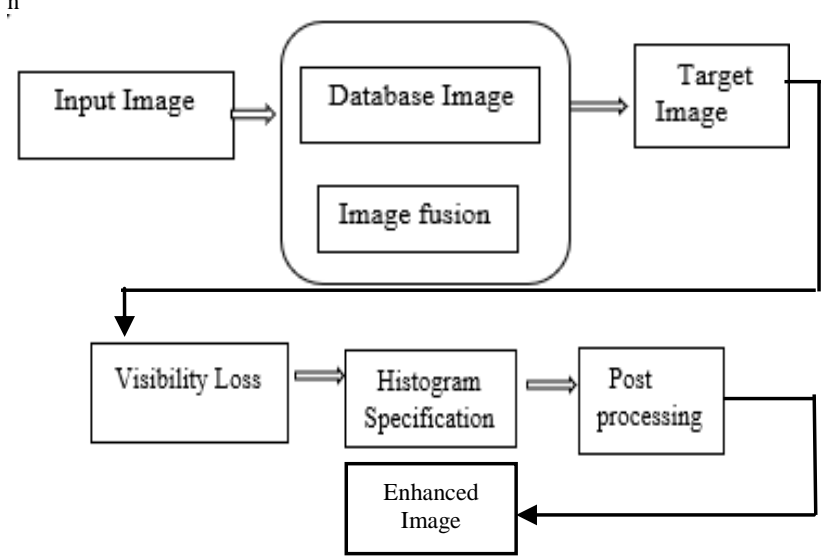

Fig.1 General Framework of Proposed Image Enhancement Method

The entire process is divided into four stages

3.1 Target Image

3.2 Image Fusion

3.3 Histogram specification

3.4 Post processing

\subsection{Target Image}

Target Image phase with an automated way of Generation phase and target histogram. It consist of following stage:

\subsubsection{Image selection from database}

Let $\mathrm{H} 1$ and $\mathrm{H} 2$ represent the histogram of the grayscale versions of $f$ and fd respectively of same size. The histograms $\mathrm{H} 1$ and $\mathrm{H} 2$ has the correlation between them can be obtained as follows.

where

$\mathrm{d}\left(\mathrm{H}_{1}, \mathrm{H}_{2}\right)=\frac{\sum_{\mathrm{i}}\left(\mathrm{H}_{1}(\mathrm{i})-\overline{\mathrm{H}}_{1}\right)\left(\mathrm{H}_{2}(\mathrm{i})-\overline{\mathrm{H}}_{2}\right)}{\sqrt{\left(\sum_{\mathrm{i}}\left(\mathrm{H}_{1}(\mathrm{i})-\overline{\mathrm{H}}_{1}\right)\right)^{2}\left(\sum_{\mathrm{i}}\left(\mathrm{H}_{2}(\mathrm{i})-\overline{\mathrm{H}}_{2}\right)\right)^{2}}} \forall_{\mathrm{i}} \in$
$\{1,2, \ldots, 255\}$

where $\overline{\mathrm{H}}_{\mathrm{k}}=\frac{1}{\mathrm{~N}} \sum_{\mathrm{i}} \mathrm{H}_{\mathrm{k}}(\mathrm{I})$

Let $\mathrm{I}=\{\mathrm{I} 1, \mathrm{I} 2 \ldots \ldots . . \mathrm{In}\}$ representa the retrieved set of highly correlated images from the database. statistical parameters such as mean, entropy, visibility extracted from $\mathrm{f}$ and $\mathrm{I}$.

$\operatorname{Mean}(\mu)$ represents the average Intensity value of an image.

$\mu=\frac{1}{\mathrm{MN}} \sum_{\mathrm{m}=1}^{\mathrm{M}} \sum_{\mathrm{n}=1}^{\mathrm{N}} \mathrm{f}_{\mathrm{m}, \mathrm{n}}$

Where fm,n represents image pixel value and $\mathrm{MN}$ represent the total number of pixels in the Image.

Entopy(q) measures the information content in an image

$\operatorname{Entropy}(\mathrm{q})=-\sum_{\mathrm{i}=0}^{255} \mathrm{P}(\mathrm{i}) \log _{2} \mathrm{P}(\mathrm{i})$

Where $\mathrm{p}(\mathrm{i})$ is the probability density function(PDF).

Visibility $(\mathrm{V})$ can be defined as a measure of how clearly an image can be seen or viewed.

$$
V=\sum_{m=1}^{M} \sum_{n=1}^{N}\left|f_{m, n}-\left(\frac{\mu}{\mu^{\alpha+1}}\right)\right|
$$

Where fm,n represent image pixel value, $\mu$ represent mean intensity value and $\infty$ is a visual constant value from 0.6 to 0.7 .

Euclidean Distance, $\mathrm{d}\left(\mathrm{x}_{\mathrm{f}}, \mathrm{x}_{\mathrm{d}}\right)=\left\|\mathrm{x}_{\mathrm{f}}-\mathrm{x}_{\mathrm{d}}\right\|^{2}$

Where $x f=[\mu f, E f, v f] \& x d=[\mu d, E d, v d]$ represent computed mean, entropy \& visibility of input image and database image I. Discussed by Raji.et al.

\subsection{Image Fusion}

The Image Fusion technique is used to obtain a target image. It is the process of combining two or more images such that the resultant image will be having information from the entire source images selected for fusion. In the proposed FHN method Image Fusion is done based on entropy. A new set of images can be obtained by fusing all combinations of images in I'.

Where I' set of selected images for fusion.

I'CI,E $=\{\mathrm{e} 1, \mathrm{e} 2, \ldots \mathrm{e} 5\}$ represent the entropy of all images inI'.Images selected from I' to be fused and e1,e2 represent their entropy.value of parameters B can be based on the following conditions.

$$
\beta=\left\{\begin{array}{rc}
0.25, & e_{1}<e_{2} \\
0.75, & e_{1}>e_{2} \\
0.5, & e_{1}=e_{2}
\end{array}\right.
$$

Let $\mathrm{xm}, \mathrm{n}, \mathrm{ym}, \mathrm{n}$ represent pixel values of images to be fused and $\mathrm{zm}, \mathrm{n}$ represent pixel values of the fused image.

$$
Z_{m, n}=\beta\left(X_{m, n}\right)+(1-\beta)\left(Y_{m, n}\right)
$$

$Z=\{z 1, z 2 \ldots \ldots \ldots z 10\}$ be the set of fused images. The one with the highest entropy is selected as target image( $\left.f^{\prime}\right)$. Discussed by Raji et al.

\section{Visibility Loss:}

The Selected target histogram is unsuitable for the Input Image and histogram specification of Input image to target Image would result in loss of visibility rather than enhancement. We refer to this problem as visibility loss .Emprically it is found that the successful enhancement EME of target image selected must be greater than EME of input 
image so that histogram specification would result in effective enhancement. If the condition is not satisfied, histogram specification would lead to visibility loss problem.

\subsection{Histogram Specification.}

The purpose of histogram specification(HS) is to get a desirable histogram. let $\mathrm{h}_{\mathrm{fc}}, \mathrm{h}_{\mathrm{f}}$ c represents the histograms of $f_{c}$ and $f_{c}^{\prime}$ respectively for a particular color channel $c$. The cumulative distribution functions $\mathrm{F}_{\mathrm{fc}}$ and $\mathrm{F}_{\mathrm{fc}}$ for $\mathrm{h}_{\mathrm{fc}}$ and $\mathrm{h}_{\mathrm{f}}$ can be obtained as,

$$
\begin{aligned}
& \mathrm{F}_{\mathrm{f}_{\mathrm{c}}}=\sum_{\mathrm{i}=0}^{\mathrm{j}} \mathrm{h}_{\mathrm{f}_{\mathrm{c}}}(\mathrm{i}) \quad \mathrm{j} \in\{0,1 \ldots \ldots . . \mathrm{L}-1)(8) \\
& \mathrm{F}_{\mathrm{f}_{\mathrm{c}}}=\sum_{\mathrm{i}=0}^{\mathrm{j}} \mathrm{h}_{\mathrm{f}_{\mathrm{c}}}(\mathrm{i})
\end{aligned}
$$

A look-up table can be designed by looking for grey level match.for each gray level match.For each gray level $h_{f c}(1) E\{0,1, \ldots . L-1\} h_{f^{\prime} c}(j)$. such that $F_{f^{\prime} c}(j)$ best matches $F_{f c}(1)$.

$$
\operatorname{lookup}_{\mathrm{c}}(1)=\mathrm{j}
$$

$f_{c}$ is obtained by replacing each pixel in $f_{c}$ with value $j$,

$\mathrm{f}_{\mathrm{c}}(\mathrm{m}, \mathrm{n})=\operatorname{lookup}_{\mathrm{c}}\left(\mathrm{f}_{\mathrm{c}}(\mathrm{m}, \mathrm{n})\right)$

Discussed by Jayaraman.

\subsection{Post Processing}

The post processing phase makes the images visually clearer.Here post processing is done using hyperbolic tangent function or tanh.It is a non-linear function. The range of tanh function is from -1 to +1 .tanh is also sigmoidal(s-shaped).tanh functions are used in feed-forward networks.

$$
\tanh (x)=\frac{e^{x}-e^{-x}}{e^{x}+e^{-x}}
$$

tanh is also like logistic sigmoid but better. The range of the tanh function is from (-1 to 1$)$. tanh is also sigmoidal (s shaped).

The advantage is that the negative inputs will be mapped strongly negative and the zero inputs will be mapped near zero in the tanh graph.

- The function is differentiable.

- The function is monotonic while its derivative is not monotonic.

- The tanh function is mainly used classification between two classes.

- Both tanh and logistic sigmoid activation functions are used in feed-forward nets.

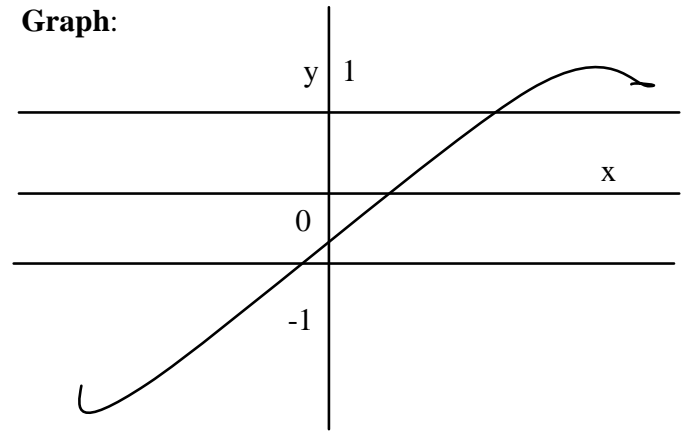

$Y=\tanh x$

\section{ALGORITHM}

The following are the steps involved in the color Image Enhancement technique of fusion using automated Histogram Specification.

Step1:Image is selected from the database is taken as the Input Image for the system to process.

Step2:The Fusion of Images leads to the Generation of target Image.

Step3:Finding the visibility loss in images has done in validation of target image phase.

Step4:Using Histogram specification method to minimize the visibility loss.

Step5:Applying Post Processing technique for an enhanced Image as a resultant Image.

\section{EXPERIMENTAL RESULTS}

To obtain maximum enhancement we have used different categories of colour images which is shown in fig. 2 sample set of images in the database. In fig. 3 we have generated different set of Image fusion. In fig.4, fig.5, fig. 6 we have shown the input images, fusion of an images, enhanced Image of proposed method of FHN method and Existing method R.Raji et al. method .The Result of FNH method is better compared with the R.Raji et al method.

We have used three evaluation metrics EME,AMBE and CII metrics. The fig.4,5,6 shows the experimental result obtained for three images belonging to the categories of image with color image,fog image and low contrast image respectively. From the results obtained, we can see that proposed method is capable of enhancing different categories of images since it is using the automated generation of target image to be fused and enhanced.

The values obtained for various evaluation metrics are shown in Table1. We have used three evaluation metrics EME, AMBE and CII metrics. EME values are obtained for the purpose of measure of enhancement of an image.EME is also called as measure of image enhancement or contrast measure.

The measure of enhancement is used to find the average ratio of maximum to minimum intensities in decibels. AMBE measures the deviation of the processed image mean from the input image mean. AMBE values are lower in the proposed method which indicates that brightness of the original image is preserved in the enhanced image. The values in the CII shows the significant improvement in the contrast of the enhan ${ }^{1}$ ced image. The sample set of Images and Experimental results as follows, 
Experimental Results
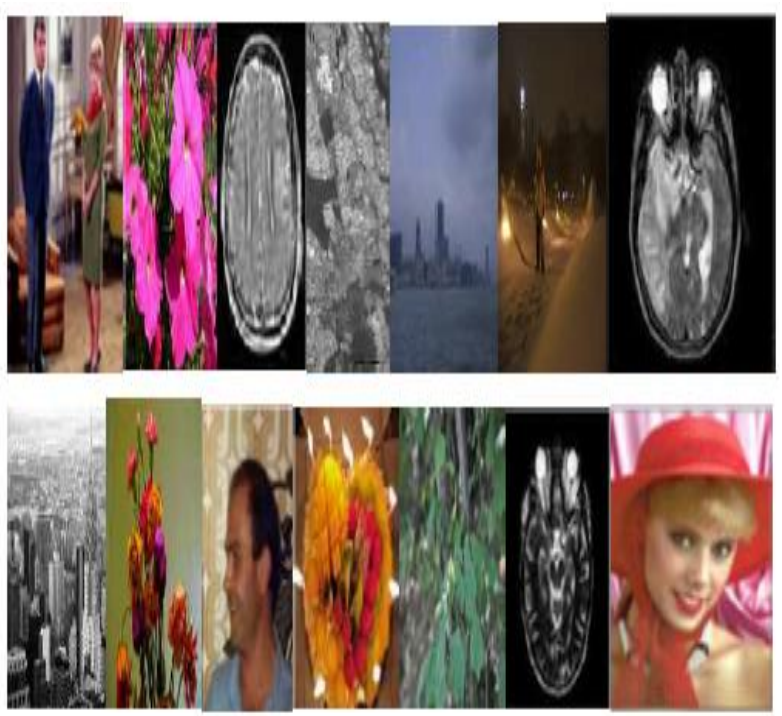

Fig.2 Sample set of images in the Database

Experimental Results

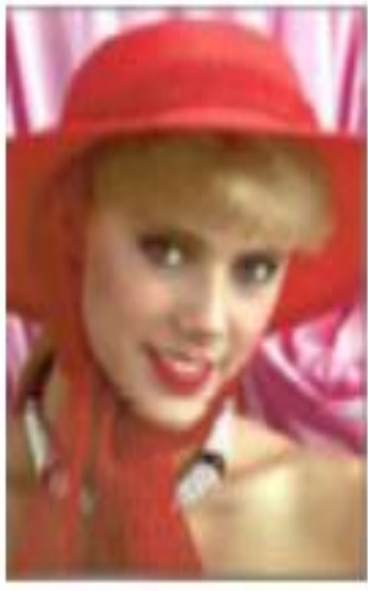

(a)

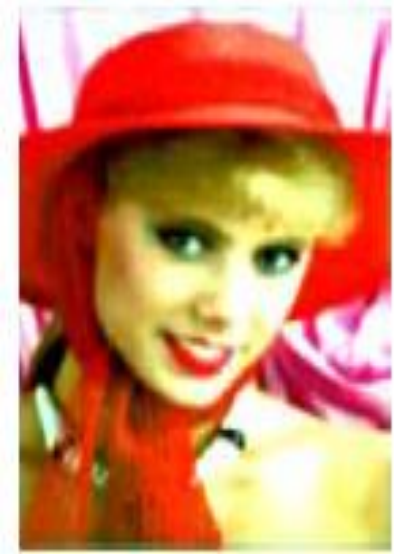

(c)
Experimental Results

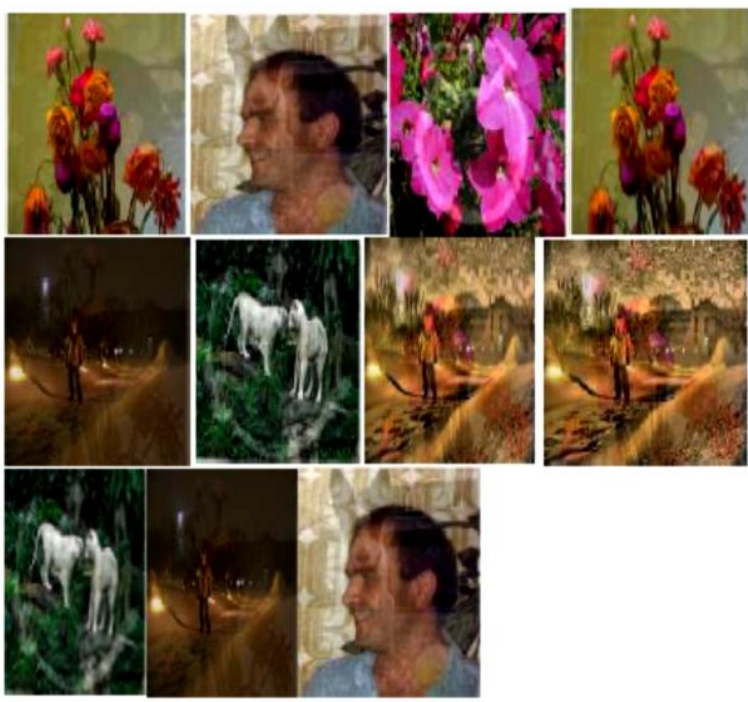

Fig.3Generated different set of Fused images

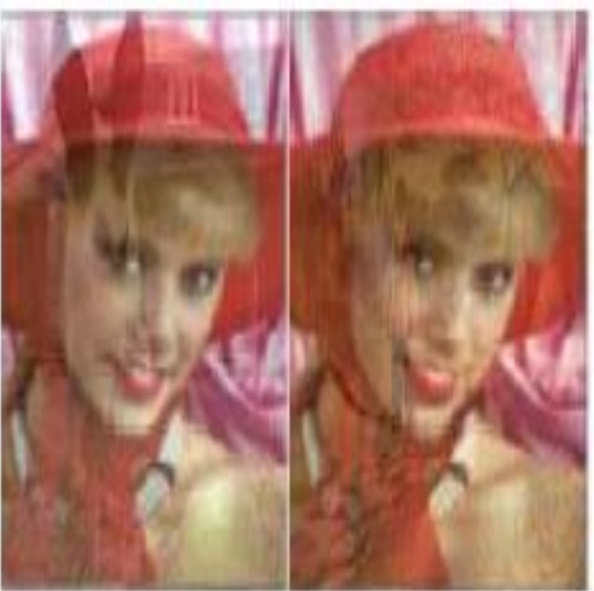

(b)

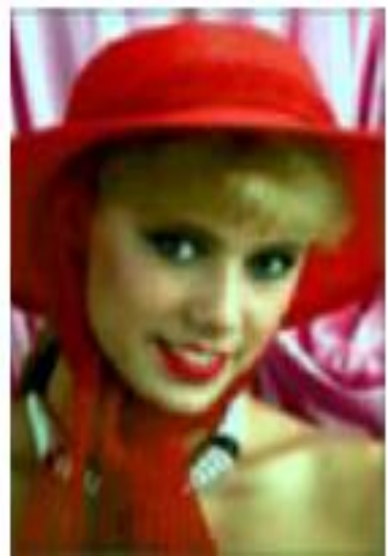

(d)

Fig.4 (a) Input Image (b) Fused image with data sets (c) Raji at el's method (d) FHN method 


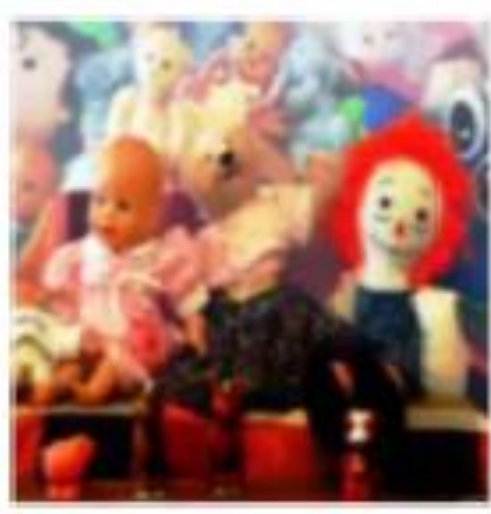

(a)

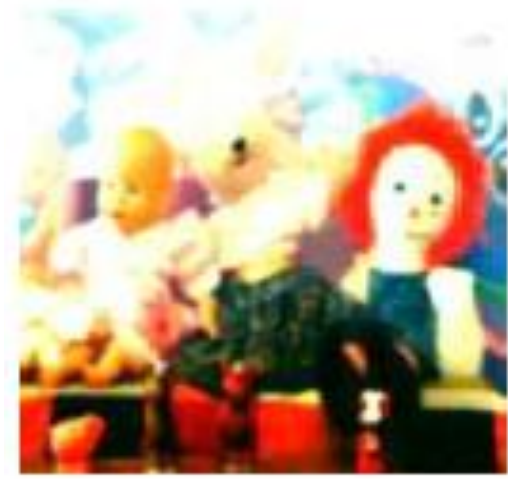

(c)

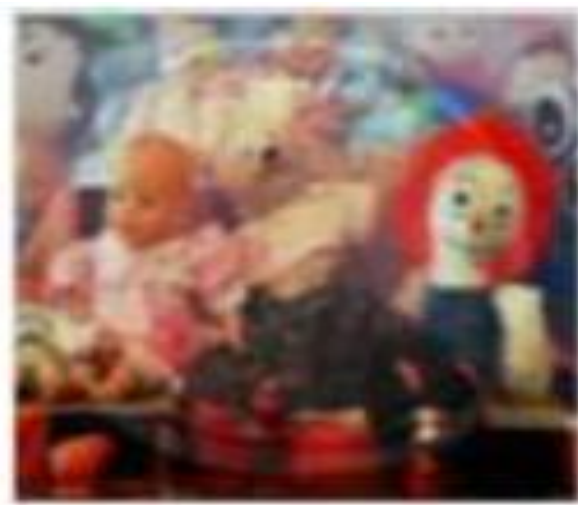

(b)

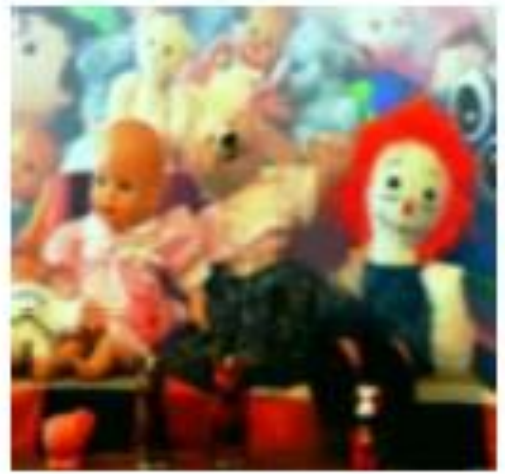

(d)

Fig.5 (a) Input Image (b) Fused image with data sets (c)Raji at el's method (d) FHN method

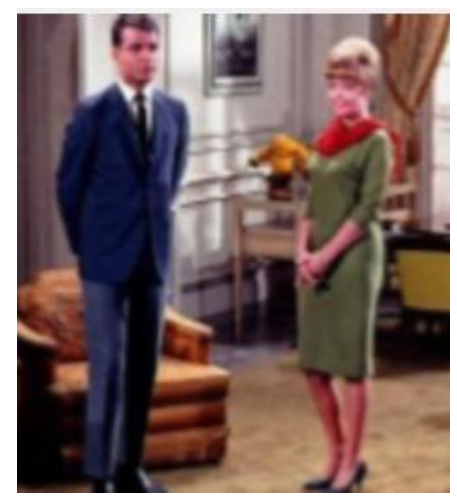

(a)

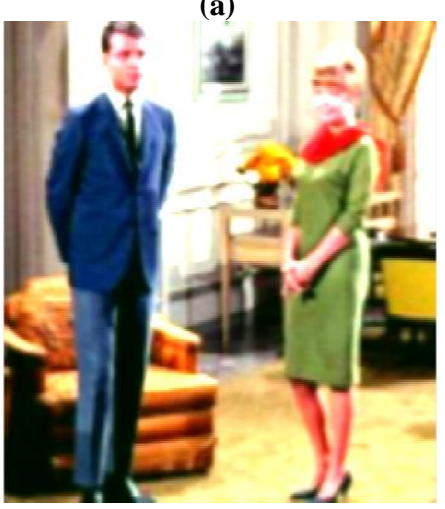

(c)

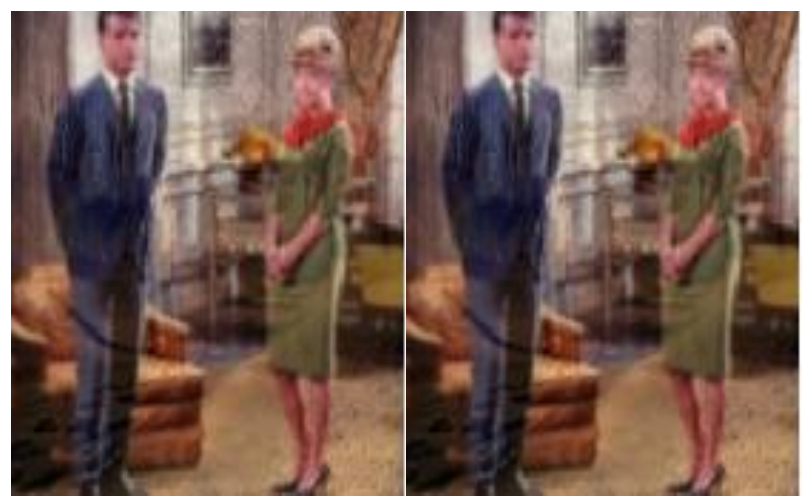

(b)

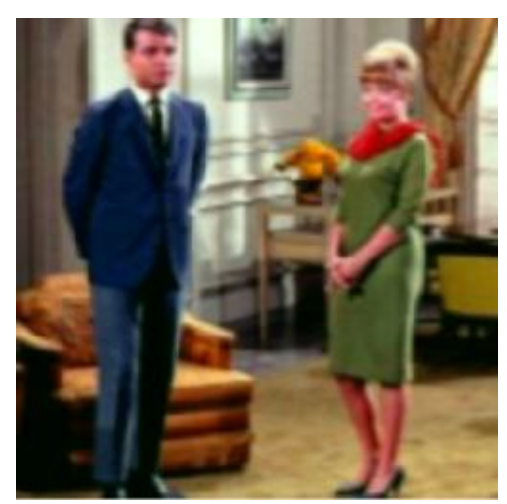

(d)

Fig.6 (a) Input Image (b) Fused Image with data sets (c) Raji at el's method (d) FHN method 


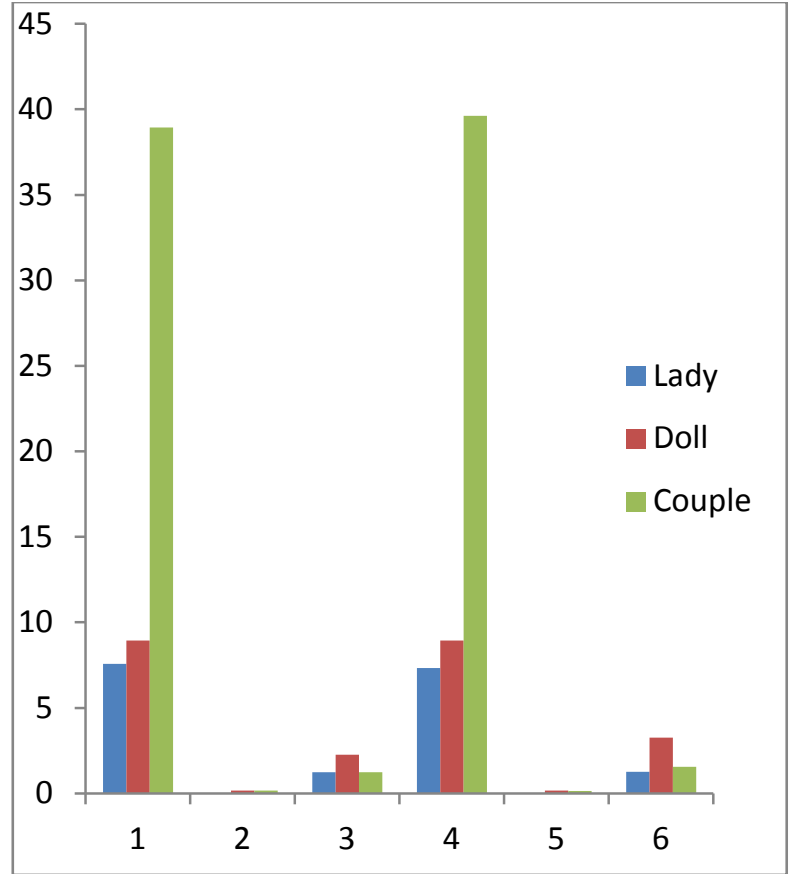

Fig.7 Graph of the proposed FHN Methods

In the table 1 . We have shown the EME, AMBE, CII Values of Images Lady, Doll \& Couple (Color, Fog, Low contrast images) EMI of FHN methods is lower compared to the Raji et al. method. The above given couple image is Low contrast image hence the value of FHN method should be higher compared to Raji et al.

The AMBE values are lower in the proposed FNH methods which indicates that brightness of the original Images is present in the enhanced image. The AMBE values are lower compared to the Raji et al. method. CII values are greater than the existing Raji et al. method. Hence the table.1 evident that FHN method gives good result compared to Raji et al's method.In Fig.7 shows the Graph of the proposed FHN method of the three images(lady,doll,couple).

\section{CONCLUSION}

This Paper explains the automated histogram specification of image fusion for color image enhancement.The proposed [FHN] method contains a new approach to detect visibility Loss problem and its solution. The post processing is done using tanh or hyperbolic tangent function to achieve the maximum enhancement. The proposed [FHN] method has been proved to be successful approach to deal with various categories of images.

\section{REFERENCES}

[1] T.zhang,R.Ramakrishnan and M.Livny,BIRCH:A New Data Clustering Algorithm and its Applications,Data Mining and Knowledge Discovery,vol,1,no,2,p.141182,june1997.
[2] Agaian Sos S,Karen panetta and Artyom M.Grigoryan,A new measure of image enhancement,in IASTED International Conference on Signal Processing \& Communication,p.19-22,September 2000.

[3] S.F.Naik and C.A.Murthy,Hue-preserving color image enhancement without gamut problem, IEEE Trans.Image process,vol.12,no.12,p.1591-1598, Dec.2003.

[4] Avcibas,N.Memon and B.Sankur,Steganalysis using Image Quality Metrics,IEEE Transactions on Image processing,vol.12,no.2,pp.221-228, February 2003.

[5] Hassan Naglaa, and Norio Akamatsu.A New Approach for Contrast Enhancement Using Sigmoid Function, The International Arab Journal of Information Technology,vol 1,No 2,p.221-225,July 2004.

[6] S.K.Saha,A.K.Das,Chanda,CBIR using perception based Texture and Color Measures, Pattern Recognition,Proceedings of the $17^{\text {th }}$ International Conferences,No.3,p.985-8,Aug.2004.

[7] M.Grundland,N.A.Dodgson,Colorhistogram specification by histogram warping,in:proceedings of the SPIE,Vol.5667,No.2,pp610-624,June 2004.

[8] yad Jafar Hao Ying,A New Method For Image Constrast Enhancement Based on Automatic Specification of local Histogram,International Journal of Computer Science and Network security,VOL.7,No.7,p.1-10,July 2007.

[9] Rafael C.Gonzalez,Richard E.Woods,Digital Image Processing $\left(3^{\text {rd }}\right.$ Edition $)$, Upper Saddle River,NJ:PrenticeHall,August 2007.

[10] Pavithra P,Ramyashree N,Shruthi T.V and Jharna Majumdar,Image Enhancement by Histogram Specification Using Multiple Target Images, Special Issue Issue 2,3,4,for International Conference,P.193200, August 2010.

[11] .Kalyan Roy and Joydeep Mukherjee,Image Similarity Measure using Color Histogram,Color Coherence Vector, and Sobel Method, International Journal of Science and Research,Volume 2 Issue 1,p.538543,January 2013.

[12] M.Nikolova,,Afast algorithm for exact histogram specification Simple extension to colour images,in SCALE Space and Variational Methods in Computer Vision. Lecture Notes in Computer Science 7893,Springer,p.174-185,February 2013.

[13] Codruta Orniana Ancuti and Cosmin Ancuti,Single Image Dehazing by Multi-Scale Fusion,IEEE Transactions on Image Processing, Vol.22, no.8,p.327132821,August 2013.

[14] Raji R,Deepak Mishra,Madhu S.Nair.A Novel Texture Based Automated Histogram Specification Using Image Fusion of International conference,No. 46,p.15011509,2015 . 